\title{
Progressive Supranuclear Palsy: A Review of Co-existing Neurodegeneration
}

\author{
J Keith-Rokosh, LC Ang
}

\begin{abstract}
Objectives: The neuropathological findings of 32 progressive supranuclear palsy (PSP) cases over a period of 17 years were reviewed. Results: Of the 26 cases with adequate clinical data, 20 patients either presented with cognitive dysfunction or developed a cognitive impairment subsequently during the course of the disease. Co-existing changes of argyrophilic grains and corticobasal degeneration (CBD) were found in $28 \%$ and $32 \%$ of the cases respectively. Alzheimer-related pathology was found in $69 \%$ of cases but only $18.75 \%$ of cases fulfilled the consortium to establish a registry for Alzheimer's disease (CERAD) criteria for either definite or probable Alzheimer's disease. Lewy bodies were noted in four cases $(12.5 \%)$, all in the subcortical regions. Only seven cases of PSP showed no pathological evidence of other co-existing neurodegenerative diseases. The severity of the cerebrovascular pathology in this cohort was insufficient to explain any clinical symptomatology. Conclusions: As in previous studies, this study has demonstrated the frequent co-existence of pathological changes usually noted in other neurodegenerative diseases in PSP. Whether these coexisting pathological changes contribute to the cognitive impairment in PSP remains uncertain.
\end{abstract}

RÉSUMÉ: Paralysie supranucléaire progressive et neurodégénérescence coexistante. Objectifs : Les observations neuropathologiques provenant de 32 patients atteints de paralysie supranucléaire progressive (PSP) au cours d'une période de 17 ans ont été révisées. Résultats : Vingt patients parmi les 26 pour qui les données cliniques étaient adéquates ont consulté pour une dysfonction cognitive ou ont présenté une atteinte cognitive au cours de l'évolution de la maladie. Des changements au niveau des granules argentophiles et une dégénérescence corticobasale ont été observés chez $28 \%$ et $32 \%$ des patients respectivement. Une pathologie de type Alzheimer a été observée chez $69 \%$ des patients bien que seulement 18,75\% des patients rencontraient les critères du CERAD pour une maladie d'Alzheimer certaine ou probable. Des corps de Lewy ont été observés chez quatre patients $(12,5 \%)$, exclusivement dans les régions sous corticales. Seulement sept cas de PSP n'avaient aucune manifestation anatomopathologique d'une autre maladie neurodégénérative coexistante. La sévérité de la pathologie cérébrovasculaire dans cette cohorte était insuffisante pour expliquer la symptomatologie clinique. Conclusions : Cette étude, comme les études antérieures, démontre la coexistence fréquente dans la PSP de changements anatomopathologiques présents dans d'autres maladies neurodégénératives. La contribution de ces changements anatomopathologiques au déficit cognitif dans la PSP demeure obscure.

Can. J. Neurol. Sci. 2008; 35: 602-608

Progressive supranuclear palsy (PSP) is an uncommon neurodegenerative disease, with reported prevalence rates varying from 1.3 per $100,000^{1}$ to 6.4 per $100,000 .^{2}$ This disease was first described as a clinico-pathological entity by Steele, Richardson and Olzsewski from the University of Toronto. ${ }^{3}$ They reported nine patients with a progressive neurological disorder characterized by downward gaze palsy, pseudobulbar palsy, dysarthria, dystonic rigidity of neck and upper trunk with inconstant cerebellar and pyramidal symptoms. In seven patients, neuropathological examination including microscopic evaluation was performed. Progressive supranuclear palsy is also clinically categorized as a Parkinson plus syndrome, and typically has an insidious onset around the sixth decade of life. Falls and postural instability are the common early presentations. Symptoms that later emerge include axial rigidity,

From the Department of Pathology, London Health Sciences Centre and University of Western Ontario, London, Ontario, Canada.

Received March 28, 2008. Final Revisions Submitted May 26, 2008. Correspondence to: Lee Cyn Ang, Department of Pathology, London Health Sciences Centre, University Hospital, 339 Windermere Rd., P.O. Box 5339, London, Ontario, N6A 5A5, Canada. 
bradykinesia, impaired gait, and downward gaze palsy. Neuropsychiatric features such as cognitive dysfunction and behavioural symptoms can also occur. ${ }^{4}$

Attempts have been made to develop clinical criteria for the diagnosis of PSP, ${ }^{5}$ yet clinical overlap between PSP, corticobasal degeneration (CBD) and frontotemporal lobar degeneration (FTLD) is being increasingly recognized ${ }^{6}$ There are currently no accepted useful ancillary studies aiding clinicians in confirming this diagnosis, and postmortem confirmation by neuropathological examination is necessary in most instances. The pathology of PSP is characterized by the accumulation of abnormal tau protein within neurons (neurofibrillary tangles) and glial cells (tufted astrocytes, thorny astrocytes and coiled bodies) in the brain. ${ }^{4}$ The distribution of tau inclusions is mainly subcortical and the globus pallidus, subthalamic nucleus, and substantia nigra, which are severely affected in most cases. Other subcortical nuclei are affected to varying degrees, and the cerebral cortex, such as the parasagittal frontal gyrus, can also show the characteristic tau inclusions ${ }^{4,7}$ Cases of severe cortical degeneration in PSP have been described. ${ }^{8}$

Pathological heterogeneity is common in PSP, and there is significant overlap between the histopathology of PSP and other tauopathies such as CBD and FTLD with tau-positive inclusions. Co-existing neurodegenerative diseases such as Alzheimer's disease (AD), Lewy body disease (LB), argyrophilic grains (AG) and Pick's disease have all been described. In this retrospective study, we reviewed our neuropathological experience with PSP from a tertiary centre in Southwestern Ontario, Canada over a period of 17 years.

\section{Materials AND Methods}

All autopsy cases $(\mathrm{N}=44)$ with a pathological diagnosis of 'progressive supranuclear palsy' $(\mathrm{N}=35)$, 'tauopathy with features of progressive supranuclear palsy and corticobasal degeneration' $(\mathrm{N}=2)$, 'unspecified tauopathy' $(\mathrm{N}=2)$, and 'corticobasal degeneration' $(\mathrm{N}=5)$ performed at the Department of Pathology, London Health Sciences Centre, Canada since 1991 were retrieved for review. The project was approved by Research Ethics Board of the University of Western Ontario and Archival Tissue Committee, Department of Pathology, London Health Sciences Centre. The slides prepared at the time of autopsy were reviewed to confirm or refute the diagnosis of PSP. Two cases were excluded as the slides were not available for review. The five cases that were initially diagnosed histologically as CBD were confirmed to be CBD with no PSP features and were excluded from further analysis. Based on the typical and distinguishing features of PSP listed in the ISN text on Neurodegenerative diseases ${ }^{4}$ and the Preliminary NINDS neuropathological criteria for PSP, ${ }^{9}$ five cases were excluded from the study as the authors felt that an alternative diagnosis was more appropriate. Two of the excluded cases were classified as 'unspecified multisystem tauopathy', characterized by severe cortical atrophy with gliosis, widespread tau inclusions (including neurofibrillary tangles and coiled bodies, and lesser numbers of thorny astrocytes) and sparing of the subcortical areas that are usually severely affected in PSP (such as globus pallidus, pontine nuclei, subthalamic nucleus and substantia nigra). One case originally called PSP was reclassified as CBD with no PSP features, as there was a relative abundance of ballooned neurons and glial plaques. A case was reclassified as FTLD with tau-positive inclusions, and another case was reclassified as multisystem atrophy. The final number of cases included in this study was 32 .

The clinical records of each case were reviewed. The gender, age at presentation and disease duration were documented. In 26 cases information concerning the patient's cognitive status was available, and these patients were classified as those who were 'cognitively dysfunctional' (either at presentation or developing later in their disease course, $\mathrm{N}=20$ ) versus those who remained 'cognitively intact' throughout $(\mathrm{N}=6)$. In the remaining cases $(\mathrm{N}=6)$, there was insufficient data on cognitive status. The classification of 'cognitively dysfunctional' was determined when the clinical record made note of 'significant memory loss' or 'cognitive decline' or 'significant behavioural dysfunction'; no specific score on Mini Mental Status Examination was sought, and only three of the cases had detailed neuropsychological testing. Of these 20 cases, 12 had significant cognitive dysfunction at early onset (within the first year) of their disease. In the other eight cases, cognitive dysfunction developed subsequently during the course of the disease.

In 17 of the 32 cases the brain tissue had been stored in $20 \%$ neutral buffered formalin following the autopsy. For these cases with available gross tissue, the brains were re-examined and new blocks were sampled from the following anatomical structures: cerebral cortex (superior and middle frontal gyri, superior and middle temporal gyri, anterior cingulate, calcarine corticies), caudate head (at the level of the anterior limb of the internal capsule), basal ganglia at the level of the anterior commissure, thalamus (both at the level of the mamillary bodies and the subthalamic nucleus), hippocampus, amygdala, midbrain, pons (with locus ceruleus), medulla and cerebellar dentate nucleus.

When gross material was not available (13 cases), paraffin embedded tissue blocks prepared at the time of gross brain dissection were used. In two cases, paraffin embedded blocks were not available and paraffin sections cut and stained at the time of original neuropathological examination were reexamined.

The paraffin embedded blocks were cut into four micron sections and stained with Bielschowsky's silver impregnation, haematoxylin-eosin (H\&E), luxol fast blue with haematoxylineosin (H\&E/LFB), and Congo red. Immunohistochemistry was performed following the Avidin-Biotin-Complex method with the primary antibodies for phosphorylated tau (AT8, Pierce Endogen, 1:100), alpha-synuclein (Zymed, 1:50), and glial fibrillary acidic protein (GFAP, Dako, 1:500) and incubated at room temperature for 60 minutes. For the phosphorylated-tau (AT8) immunohistochemistry, antigen retrieval with a citrate buffer and the microwave was used.

\section{Co-existing Neurodegenerative Diseases}

Sections treated with modified Bielschowsky's silver technique were used to screen for Alzheimer's related changes. The frequency of neurofibrillary tangles within Ammon's horn, the entorrhinal cortex, subiculum, striate and parastriate corticies were graded as none $(0)$, infrequent (i), moderate (ii), frequent (iii) and ghost (g) as per the Braak and Braak staging system, ${ }^{10,11}$ and a pathological stage from 0 through 6 was assigned. The frequency of neuritic plaques was assessed and an age-related 
plaque density score and diagnostic group were generated using the consortium to establish a registry for Alzheimer's disease (CERAD) staging system for Alzheimer's disease. ${ }^{12}$ Phosphorylated-tau immunohistochemistry was also used to examine Alzheimer's type pathology. ${ }^{11}$

A thorough search for Lewy bodies was undertaken using $\mathrm{H} \& \mathrm{E}$ sections and alpha-synuclein immunostained sections from the substantia nigra, locus ceruleus, dorsal motor nucleus of the vagus, nucleus basalis, amygdala and anterior cingulate cortex. Argyrophilic grains were sought by examining phosphorylatedtau immunostained sections of the hippocampus, amygdala, entorhinal, cingulate, and insular cortices.

\section{RESULTS \\ Clinical Data}

Of the 32 cases included in the analysis, 19 were males and 13 were females, with an average age of 73.35 years at the time of death (range 55 to 85 years). The disease duration ranged from 1.5 to 11 years with a median of 5.22 years (see Table). Twenty cases were cognitively dysfunctional, six remained cognitively intact, and no clinical information regarding cognitive status was available in the other six cases. There was no difference in patient age or disease duration between the group who was cognitively dysfunctional versus those cognitively intact $(\mathrm{p}=$ $0.272, \mathrm{p}=0.453$ respectively). The majority of the 32 patients carried a clinical diagnosis of PSP (15 cases, including Case 19 labeled as PSP/CBD). Five patients were clinically labeled as Alzheimer's disease (Cases 7, 22, 23, 24 and 27), two were labeled as vascular dementia (Cases 21 and 32), two were labeled as 'functional decline' (Cases 26 and 29) and the following clinical labels were applied to a single case each: Pick's disease (Case 18), Parkinson's disease/Alzheimer's disease (Case 5), Parkinson's disease (PD) (Case 16), ALS (Case 2 ), cerebellar degeneration (Case 15) and primary progressive aphasia (Case 28). Clinical diagnosis for the remaining two cases was not available (Cases 1, and 25).

\section{Pathology}

Co-existing neurodegenerative phenomena are presented in the Table.

Alzheimer's related changes frequently co-existed with PSP and were found in 22 of the 32 cases (69\%). The severity of the Alzheimer pathology ranged from Braak and Braak stage 1 (three cases), stage 2 (eight cases), stage 3 (six cases), stage 4 (four cases), to stage 5 (one case). There was a significant correlation between Braak and Braak Alzheimer's stage and age (correlation coefficient $0.49, \mathrm{p}=0.01)$. Only six cases $(18.75 \%$ ) fulfilled the CERAD criteria for either definite or probable Alzheimer's disease and all these cases had clinical history of cognitive dysfunction.

Argyrophilic grains (Figure 1) were found in the mesial temporal lobe, especially the amygdala, in nine cases $(28 \%)$, and all of these cases also contained ballooned neurons. All nine cases with argyrophilc grains were cognitively impaired.

Ten PSP cases (32\%) had overlapping 'CBD features', as seen in Figure 2A and 2B, defined in this study as both tauimmunopositive glial plaques (Figure 2A) and ballooned neurons (Figure 2B) not limited to a limbic distribution. Nine of the ten cases were cognitively impaired. Six of these ten cases also had co-existing argyrophilic grains.

Lewy bodies, as defined as round, eosinophilic cytoplasmic inclusions, with or without a halo, visible on H\&E (Figure 3A) and immunolabelling with alpha-synuclein (Figure $3 \mathrm{~B}$ ), were present in four cases in the following distribution: Case 13 contained Lewy bodies in the substantia nigra and dorsal motor nucleus of the vagus, Case 19 contained Lewy bodies only in the dorsal motor nucleus of the vagus, Case 24 in the substantia nigra and nucleus basalis, Case 28 in the amygdala, and Case 32 contained Lewy bodies in the locus ceruleus, nucleus basalis, dorsal motor nucleus of the vagus and substantia nigra.

There were five cases with brain infarcts. Four of these patients were cognitively impaired, (cognitive status is not available for Case 3) but the sizes and regional localization of these infarcts (basal ganglionic, cerebellar) could not explain the cognitive impairment. In Case 33, the infarcts were more widespread (occipital cortex, centrum semiovale, internal capsule and pons). Congo red staining or beta-amyloid immunolabelling was performed in these cases, and in a single case amyloid angiopathy was noted (but there were no associated cerebral infarcts or hemorrhage). In one case clinically labeled as vascular dementia (Case 21), no infarcts were found.

Twelve cases had changes of three or more neurodegenerative processes on pathological examination. One case had PSP, CBD features, argyrophilic grains and Lewy bodies (Case 24), two cases had combined PSP, CBD features and Lewy bodies (Case 13 and 28), two cases had PSP, Lewy bodies and argyrophilic grains; as mentioned above six cases had combined PSP, CBD and argyrophilic grains, and a single case had PSP, definite AD, CBD features and argyrophilic grains (Case 7).

In our series only seven cases had pathological findings of 'pure' PSP without any co-existing neurodegenerative phenomena. Three of these seven cases were cognitively intact,

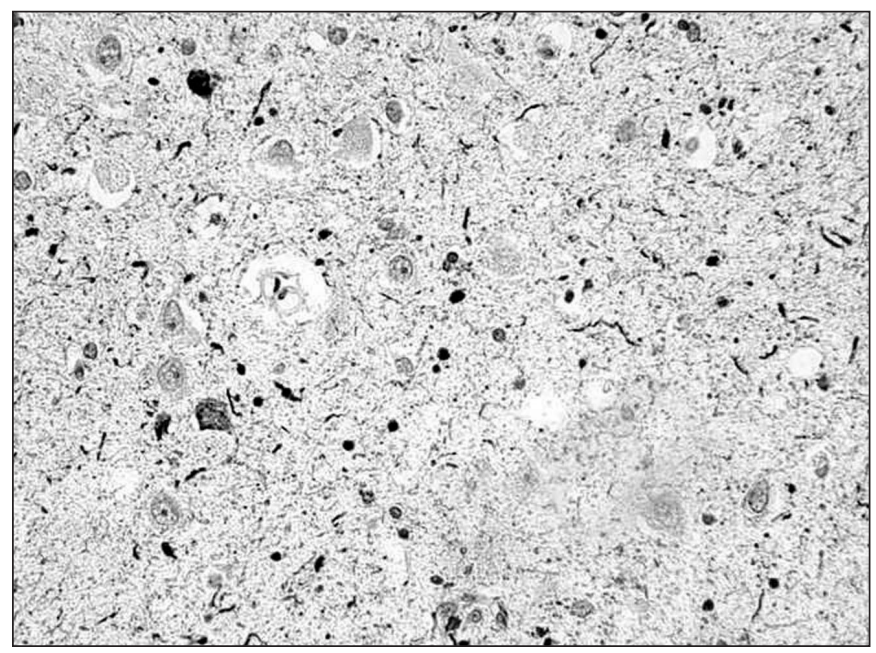

Figure 1: Short, coarse phosphorylated tau-immunopositive grains within the mesial temporal lobe (AT8, original magnification X 400). 
Table: Clinical information, cognitive status and co-existing neurodegenerative phenomena

\begin{tabular}{|c|c|c|c|c|c|c|c|c|c|c|c|c|}
\hline \# & $\begin{array}{l}\text { Clin } \\
\text { Dx }\end{array}$ & $\begin{array}{l}\text { Cog } \\
\text { Status }\end{array}$ & Sex & $\begin{array}{l}\text { Age @ } \\
\text { death } \\
\text { (yrs) }\end{array}$ & $\begin{array}{l}\text { Disease } \\
\text { duration } \\
\text { (yrs) }\end{array}$ & $\begin{array}{l}\text { AD } \\
\text { Stage } \\
\text { (Braak) }\end{array}$ & $\begin{array}{l}\text { CERAD } \\
\text { Group }\end{array}$ & $\mathrm{AG}$ & CVD & $\begin{array}{l}\text { Glial } \\
\text { Plaques }\end{array}$ & $\begin{array}{l}\text { Ballooned } \\
\text { Neurons }\end{array}$ & LB \\
\hline 1 & N/A & I & M & 83 & 11 & 3 & Possible & - & - & - & - & - \\
\hline 2 & ALS & I & $\mathrm{F}$ & 59 & 4 & None & Normal & - & - & - & + & - \\
\hline 3 & PSP & $\mathrm{N} / \mathrm{A}$ & $\mathrm{F}$ & 84 & $\mathrm{~N} / \mathrm{A}$ & None & Normal & - & Infarct & - & - & - \\
\hline 4 & PSP & $\mathrm{D}$ & $M$ & 76 & 5 & 3 & Probable & - & - & - & - & - \\
\hline 5 & $\mathrm{PD} / \mathrm{AD}$ & $\mathrm{D}$ & $\mathrm{F}$ & 82 & 8 & 1 & Normal & - & Infarct & - & + & - \\
\hline 6 & PSP & $\mathrm{N} / \mathrm{A}$ & $\mathrm{M}$ & 62 & $\mathrm{~N} / \mathrm{A}$ & None & Normal & - & - & - & + & - \\
\hline 7 & $\mathrm{AD}$ & $\mathrm{D}$ & $M$ & 77 & 5 & 4 & Definite & + & - & + & + & - \\
\hline 8 & PSP & $\mathrm{D}$ & $M$ & 74 & 5 & 4 & Definite & - & - & - & - & - \\
\hline 9 & PSP & $\mathrm{D}$ & $\mathrm{F}$ & 63 & 2 & 1 & Normal & - & - & + & + & - \\
\hline 10 & PSP & $\mathrm{D}$ & $M$ & 74 & 4 & 4 & Definite & - & - & - & - & - \\
\hline 11 & PSP & N/A & $M$ & 71 & N/A & 1 & Normal & - & - & - & - & - \\
\hline 12 & PSP & N/A & $M$ & 64 & $\mathrm{~N} / \mathrm{A}$ & 2 & Normal & - & - & - & - & - \\
\hline 13 & PSP & $\mathrm{D}$ & $\mathrm{F}$ & 55 & 2 & None & Normal & - & - & + & + & + \\
\hline 14 & PSP & I & $\mathrm{F}$ & 70 & 4 & None & Possible & - & - & + & + & - \\
\hline 15 & $\begin{array}{l}\mathrm{CB} \\
\text { Degen }\end{array}$ & I & $\mathrm{F}$ & 60 & 4 & None & Possible & - & - & - & - & - \\
\hline 16 & PD & $\mathrm{I}$ & $\mathrm{F}$ & 79 & N/A & 2 & Possible & - & - & - & + & - \\
\hline 17 & PSP & I & $M$ & 72 & 8 & None & Normal & - & - & - & + & - \\
\hline 18 & Pick's & $\mathrm{D}$ & $\mathrm{F}$ & 67 & $\mathrm{~N} / \mathrm{A}$ & 3 & Normal & + & - & + & + & - \\
\hline 19 & $\begin{array}{l}\mathrm{PSP} / \mathrm{C} \\
\mathrm{BD}\end{array}$ & $\mathrm{D}$ & F & 75 & 3 & 4 & Normal & + & - & - & + & + \\
\hline 20 & PSP & $\bar{D}$ & $\mathrm{M}$ & 70 & 8 & None & Normal & - & - & + & - & - \\
\hline 21 & Vasc d & $\mathrm{D}$ & $\mathrm{F}$ & 85 & N/A & 2 & Possible & + & - & - & + & - \\
\hline 22 & $\mathrm{AD}$ & $\mathrm{D}$ & $\mathrm{M}$ & 81 & 9 & 3 & Definite & - & Infarct & - & - & - \\
\hline 23 & $\mathrm{AD}$ & $\mathrm{D}$ & $M$ & 83 & 2 & 2 & Normal & + & - & + & + & - \\
\hline 24 & $\mathrm{AD}$ & $\mathrm{D}$ & $M$ & 77 & $\mathrm{~N} / \mathrm{A}$ & 2 & Normal & + & CAA & + & + & + \\
\hline 25 & N/A & $\mathrm{D}$ & $M$ & 78 & $\mathrm{~N} / \mathrm{A}$ & 2 & Normal & - & - & - & - & - \\
\hline 26 & $\begin{array}{l}\text { fn } \\
\text { decline }\end{array}$ & $\mathrm{D}$ & $\mathrm{M}$ & 66 & 1.5 & 2 & Normal & + & Infarct & + & + & - \\
\hline 27 & $\mathrm{AD}$ & $\mathrm{D}$ & $\mathrm{M}$ & 70 & N/A & None & Normal & - & - & - & + & - \\
\hline 28 & PPA & $\mathrm{D}$ & $\mathrm{F}$ & 84 & 8 & 5 & Probable & - & & + & + & + \\
\hline 29 & $\begin{array}{l}\text { fn } \\
\text { decline }\end{array}$ & $\mathrm{D}$ & $\mathrm{M}$ & 78 & 1 & 3 & Normal & + & - & + & + & - \\
\hline 30 & PSP & N/A & $M$ & 72 & $\mathrm{~N} / \mathrm{A}$ & 3 & Normal & - & - & - & - & - \\
\hline 31 & PSP & N/A & $\mathrm{F}$ & 74 & $\mathrm{~N} / \mathrm{A}$ & 3 & N/A & - & - & - & - & - \\
\hline 32 & Vasc d & $\mathrm{D}$ & $\mathrm{M}$ & 82 & 10 & 2 & Possible & + & Infarct & - & - & + \\
\hline
\end{tabular}

$\mathrm{D}=$ cognitively dysfunctional, $\mathrm{I}=$ cognitively intact, $\mathrm{AD}=$ Alzheimer's disease, vasc $\mathrm{d}=$ vascular dementia, $\mathrm{AGD}=$ Argyrophilic Grains, LB = Lewy bodies, CBD = Corticobasal Degeneration, PPA = Primary Progressive Aphasia, CVD = Cerebrovascular Disease, $\mathrm{fn}$ decline $=$ functional decline, $\mathrm{CB}$ degen $=$ cerebellar degeneration, N/A $=$ not available, $\mathrm{CAA}=$ cerebral amyloid angiopathy; $\mathrm{ALS}=$ Amyotrophic lateral sclerosis; $\mathrm{PD}=$ Parkinson's disease; $\mathrm{Clin} \mathrm{Dx}=\mathrm{Clinical}$ Diagnosis; $\operatorname{Cog}=$ Cognitive

three were cognitively impaired and in one case the cognitive status was unknown.

\section{Discussion}

This study highlights the difficulties in arriving at an accurate diagnosis for PSP clinically, as in only 47\% (15 of 32) of our cases the diagnosis of PSP was entertained while patients were alive and all these cases were diagnosed by neurologists specialized in movement disorders or neurodegenerative diseases. The rest of the cases with other clinical 'labels' were diagnosed by a mixed group of neurologists (including movement disorder and neurodegenerative disease specialists), internists and physicians from the community. These cases were labelled clinically as AD, PD, FTLD (Pick's disease), CBD, ALS, primary progressive aphasia and strokes. While movement disorder is often considered as major clinical presentation of PSP (18 of our cases), 20 cases have cognitive dysfunction with or without clinically diagnosed movement disorder.

Alzheimer's related pathology is a frequent finding in PSP brains. Gearing et al ${ }^{13}$ found that $46 \%$ of their PSP cases fulfilled the CERAD criteria for definite or probable Alzheimer's disease, 


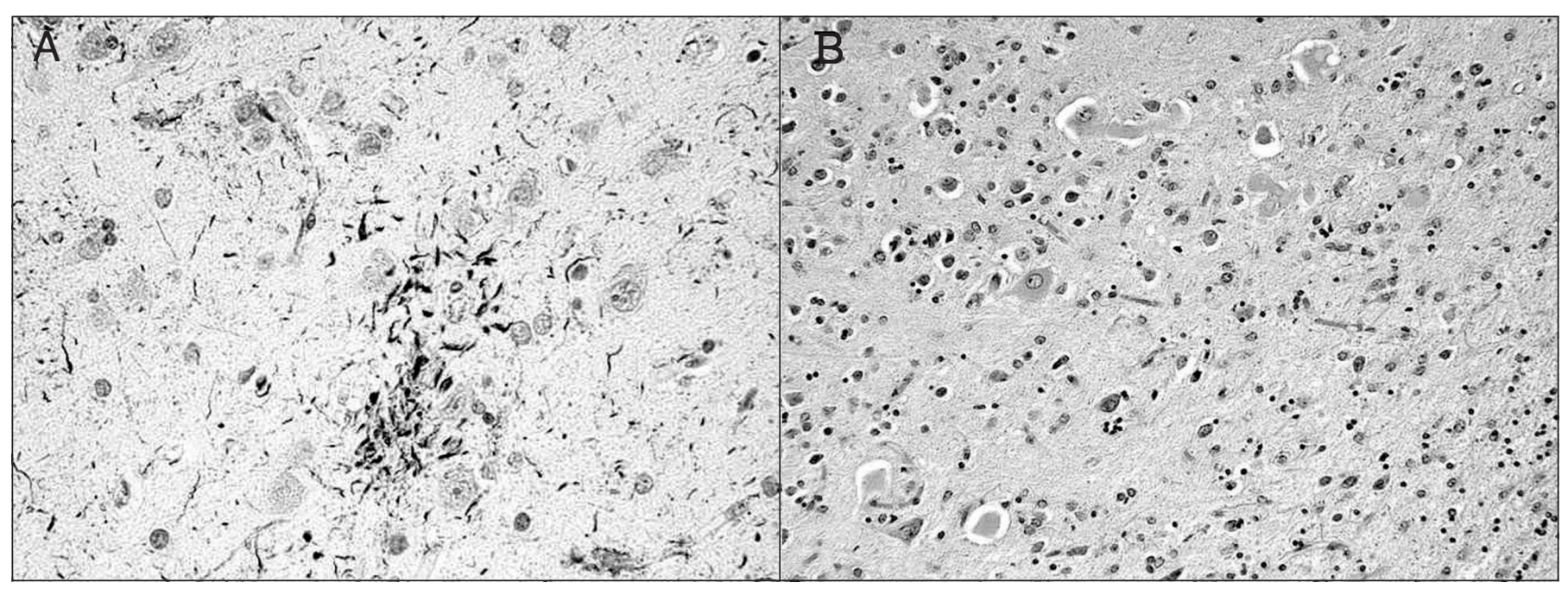

Figure 2: A) tau-immunopositive cortical glial plaque (AT8, original magnification X 200); B) Ballooned cortical neurons (H\&E X 200).

which is a greater percentage than in our patient group (18.75\%). These authors noted that the Alzheimer's pathology when coexisting with PSP was milder than that seen in typical AD cases, and they speculated that the cognitive changes seen in these patients are likely due to a combination of subcortical PSP and AD pathology as well as cortical AD pathology. ${ }^{13}$ Interestingly, all six of our patients with definite or probable Alzheimer's disease based on CERAD criteria had clinical history of cognitive dysfunction. Some problems arise when faced with a case of combined PSP and Alzheimer's disease. It can be difficult to accurately assign a Braak and Braak stage to the
Alzheimer's pathology in the presence of co-existing PSP, as some areas listed in the Braak and Braak staging system can also contain neurofibrillary tangles due to PSP. ${ }^{4,9}$ As in Gearing's study, however, we found that the neurofibrillary pathology is not as advanced in the neocortical regions as those in typical $\mathrm{AD}^{13}$ but its presence in the limbic regions could have contributed to cognitive dysfunction in a case of existing PSP that already had substantial subcortical involvement. Therefore, it is important to apply neuritic plaque density (CERAD and National Institute on Aging (NIA)-Regeant Criteria) for these cases. ${ }^{12,14}$

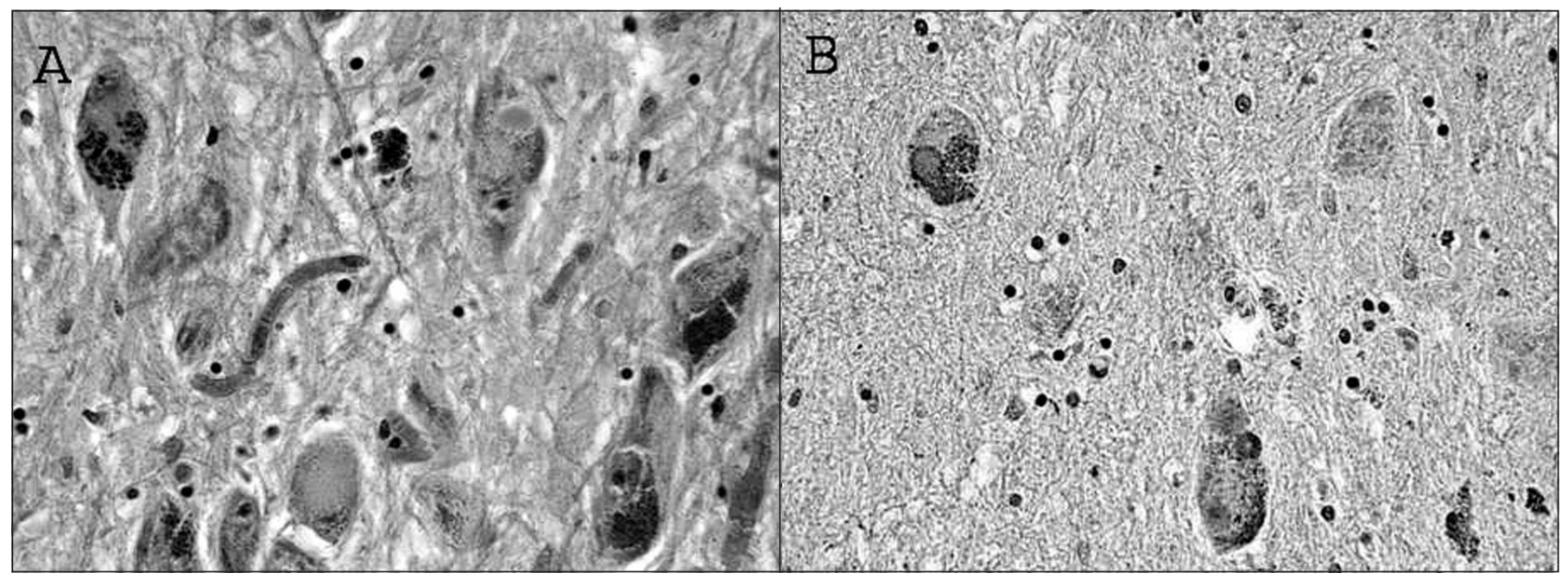

Figure 3: A) Lewy body (H\&E, original magnification X 400); B) alpha-synuclein expression within a Lewy body (alpha-synuclein, original magnification $X$ 400). 
The frequency of Lewy bodies in our PSP population is comparable to previous reports. Tsuboi et al reported Lewy bodies in the substantia nigra of PSP patients at a similar frequency as normal controls (12\% vs 9\%). ${ }^{15}$ Similarily, in Uchikado et al's series, $11 \%$ of cases from a cohort of 290 PSP patients were found to have Lewy bodies. Parkinsonism was the predominant clinical symptom in patients with combined PSP and Lewy bodies, and three out of 29 cases had dementia. ${ }^{16}$ Our experience with the distribution of Lewy bodies in PSP is similar to Uchikado's series, in that PSP cases often did not fit into the Parkinson's disease staging system by Braak and Braak. ${ }^{17}$ In $31 \%$ of Uchikado's cases with Lewy bodies there were no Lewy bodies in the substantia nigra, and/or locus ceruleus, and/or dorsal motor nucleus of the vagus, ${ }^{16}$ and in our series only one case had Lewy bodies in the substantia nigra. The absence of Lewy bodies in the substantia nigra of these PSP cases may be due to severe neuronal loss in this nucleus due to PSP per se.

The relative abundance of ballooned neurons is an important feature enabling one to distinguish between PSP and CBD on histopathology. However, several groups have reported pathologic overlap between these two entities ${ }^{18}$ and ballooned neurons are known to exist in PSP cases, especially in the entorhinal, transentorhinal, frontal and cingulate cortex. ${ }^{19,20}$ Recently, it has been suggested that the ballooned neurons seen in PSP cases are usually due to co-existing AGD, ${ }^{21}$ a condition known to occur in patients with PSP. ${ }^{22}$ It has been reported that ballooned neurons were found in more than one region of the limbic system (especially the amygdala and entorhinal cortex) in all ten cases of PSP with co-existing AGD, while ballooned neurons were very sparse in the brains of PSP without coexisting AGD, and when present, were only detected in a single anatomical region. ${ }^{21}$ In our experience, ballooned neurons were a frequent finding, including those with CBD features and all cases with co-existing AGD. The distribution of the ballooned neurons in these two scenarios was different: a widespread distribution of ballooned neurons was seen in those cases with CBD features (such as glial plaques), especially in the frontal and motor cortices, while in those cases with AGD the ballooned neurons were predominantly limbic in distribution. In our study, there is considerable overlap between PSP and CBD (33\%) as well as PSP and AGD (25\%). In two of our cases there were ballooned neurons in the absence of both argyrophilic grains and glial plaques, but they were limited to the amygdala. In the series by Togo and Dickson, two PSP patients showed ballooned neurons without other co-existing neurodegenerative diseases, which were located in the amygdala and claustrum respectively. ${ }^{21}$

Only one-fifth $(21.8 \%)$ of our cases demonstrated pathological findings of PSP alone while the majority of the cases are complex entities with co-existence or overlap with other neurodegenerative diseases. These co-existing pathological findings could render the clinical diagnosis of PSP more difficult and complicate management plans if a future remedy becomes available.

While the clinical diagnosis for PSP, especially cases presenting with cognitive dysfunction, is difficult and often inaccurate, the pathological diagnosis of PSP is also complicated by other co-existing neurodegenerative changes. The presence of co-existing neurodegenerative phenomena, including Alzheimer's related changes, argyrophilic grains, Lewy bodies and cerebrovascular disease is common in PSP, and significant overlap exists between PSP and CBD. The role these co-existing neurodegenerative phenomena may play in the development of cognitive dysfunction in PSP patient populations is uncertain but this study highlights the fact that contributory factors for cognitive impairment in PSP may be complex and multifactorial. As in all retrospective studies, this study could not address directly the issue of dementia in PSP because of the lack of clinical information on how the cognitive functions of our patients were assessed. A prospective study with adherence to specific criteria for clinical diagnosis of dementia followed by postmortem analysis of the pathological changes based on regional localization and semi-quantitative data would be necessary to improve our understanding of dementia in PSP.

\section{ACKNOWLEDGEMENTS}

The authors thank Ms. Jansen, Newman, Reade, Faurnier, Blotz, Mackie and Bergen for their technical support. We also acknowledge Drs. M. Jog, M. Borrie, A. Kertesz, B. Rudrick, M. Strong and S.J. Kish for referring these cases to us for neuropathological examination, as well as Regional Coroner, Dr Jack Stanborough, for allowing us to include cases performed under coroner's warrant. This project received fundings from the Department of Pathology, University of Western Ontario (LCA).

\section{REFERENCES}

1. Golbe LI. The epidemiology of PSP. J Neural Transm Suppl. 1994; 42:263-73.

2. Schrag A, Ben-Shlomo Y, Quinn NP. Prevalence of PSP and Multisystem Atrophy: a cross-sectional study. Lancet. 1999; 354 (9192):1771-5.

3. Steele JC, Richardson JC, Olszewski J. Progressive supranuclear palsy. Arch Neurol. 1964; 10:333-59.

4. Hauw J, Agid Y. Progressive Supranuclear Palsy (PSP) or SteeleRichardson-Olszewski Disease. In: Dickson DW, editor. Neurodegeneration: the molecular pathology of dementia and movement disorders. Basel: ISN Neuropath Press; 2003. p. 103-14.

5. Litvan I, Grafman J, Gomez C, Chase TN. Memory impairment in patients with progressive supranuclear palsy. Arch Neurol. 1989; 46(7):765-7.

6. Josephs KA, Dickson D. Diagnostic accuracy of progressive supranuclear palsy in the Society for Progressive Supranuclear Palsy brain bank. Mov Disord. 2003; 18:1018-26.

7. Braak H, Jellinger K, Braak E, Bohl J. Allocortical neurofibrillary changes in progressive supranuclear palsy. Acta Neuropathol. $1992 ; 84: 478-83$.

8. Bergeron C, Pollanen MS, Weyer L, Lang AE. Cortical degeneration in progressive supranuclear palsy. A comparison with cortical-basal ganglionic degeneration. J Neuropathol Exp Neurol. 1997; 56(6):726-34.

9. Hauw JJ, Daniel SE, Dickson D, Horupian DS, Jellinger K, Lantos PL, et al. Preliminary NINDS neuropathologic criteria for Steele-Richardson-Olszewski syndrome (progressive supranuclear palsy). Neurology. 1994; 44:2015-19.

10. Braak H, Braak E. Neuropathological staging of Alzheimer-related changes. Acta Neuropathol. 1991; 82:239-59.

11. Braak H, Alafuzoff I, Arzberger T, Kretzschmar H, Del Tredici K. Staging of Alzheimer's disease-associated neurofibrillary tangle pathology using paraffin sections and immunocytochemistry. Acta Neuropathol. 2006; 112:389-404. 
12. Gearing M, Mirra SS, Hedreen JC, Sumi SM, Hansen LA, Heyman A. The consortium to establish a registry for Alzheimer's disease (CERAD). Part X. Neuropathology confirmation of the clinical diagnosis of Alzheimer's disease. Neurology. 1995;45:461-6.

13. Gearing $M$, Olson DA, Watts R, Mirra SC. Progressive supranuclear palsy: neuropathological and clinical heterogeneity. Neurology. 1994; 44(6):1015-24

14. Newell KL, Hyman BT, Growdon JH, Hedley-White ET. Application of the National Institute on Aging (NIA)-Regean Institute Criteria for Neuropathological Diagnosis of Alzheimer's disease. J Neuropathol Exp Neurol. 1999; 58: 1147-55.

15. Tsuboi Y, Josephs KA, Boeve BF, Litvan I, Caselli RJ, Caviness JN, et al. Increased tau burden in the corticies of progressive supranuclear palsy presenting with corticobasal syndrome. Mov Disord. 2005; 20(8):982-8.

16. Uchikado H, DelleDonne A, Ahmed Z, Dickson DW. Lewy bodies in progressive supranuclear palsy represent an independent disease process. J Neuropathol Exp Neurol. 2006; 65(4):387-95.
17. Braak H, Del Tredici K, Bratzke H, Hamm-Clement J, Sandmannkeil D, Rüb U. Staging of the intracerebral inclusion body pathology associated with idiopathic Parkinson's disease (preclinical and clinical stages). J Neurol. 2002; 249 Suppl 3: $1-5$.

18. Wakabayashi K, Takahashi H. Pathological heterogeneity in progressive supranuclear palsy and corticobasal degeneration. Neuropathology. 2004; 24:79-86.

19. Mackenzie IR, Hudson LP. Achromatic neurons in the cortex of progressive supranuclear palsy. Acta Neuropathol. 1995; 90: 615-19.

20. Mori H, Oda M. Ballooned neurons in corticobasilar degeneration and progressive supranuclear palsy. Neuropathology. 1997; $17: 248-52$

21. Togo T, Dickson DW. Ballooned neurons in progressive supranuclear palsy are usually due to concurrent argyrophilic grain disease. Acta Neuropathol. 2002; 104:53-6.

22. Martinez-Lage P, Munoz DG. Prevalence and disease associations of argyrophilic grains of Braak. J Neuropathol Exp Neurol. 1997; 56(2):157-64. 